\title{
Análise faunística de insetos associados à cultura da cana-de-açúcar, em área de transição floresta amazônica - cerrado (mata de cocal), no município de União - Piauí - Brasil ${ }^{1}$
}

\section{Faunistic analysis of the insects associated to sugar-cane production in transition area amazon rain forest - cerrado (palm jungle) União region, state of Piauí - Brazil}

\author{
Rommel Tito Pinheiro Castelo Branco ${ }^{2 *}$; Gilson Lages Fortes Portela ${ }^{2}$; \\ Ocimar de Alencar Alves Barbosa ${ }^{2}$; Paulo Roberto Ramalho Silva ${ }^{3}$; \\ Luiz Evaldo de Moura Pádua ${ }^{3}$
}

\begin{abstract}
Resumo
O presente trabalho teve como objetivo estudar a entomofauna associada ao cultivo comercial de cana-de-açúcar, no município de União, Estado do Piauí. Os insetos foram coletados com armadilha luminosa, modelo "Luiz de Queiroz", provida de lâmpada F 15 T 8 BL. As coletas foram realizadas uma vez por semana, com a armadilha sendo ligada às 18:00 horas e desligada às 6:00 horas do dia seguinte, no período de dezembro de 2006 a dezembro de 2007, totalizando 52 coletas. Foram coletadas 287 espécies, submetidas à análise faunística de dominância, freqüência, abundância e constância, com 30 espécies se sobressaindo nestes índices. Astaena sp, apresentando 3.313 indivíduos, coletados em 44 das 52 coletas, Cyclocephala sp2 com 2.415 coletados em 51 das 52 e Linepithema humile com 2.299 presentes em 18 coletas.
\end{abstract}

Palavras-chave: Armadilha luminosa, scarabaeidae, coleoptera

\begin{abstract}
The objective of the project was to study the entomofauna associated to the sugar-cane commercial cultivation in União region, state of Piauí, Brazil. The insects were collected with light traps, model "Luiz de Queiroz" supplied with fluorescent lamps, ultraviolet, model F15 T8 BL posicioned in a height of $1,5 \mathrm{~m}$, at the geographic coordinates $21^{\circ} 22^{\prime} 30^{\prime \prime} \mathrm{S}$ and $48^{\circ} 13^{\prime} 0$ " W. The traps were turned on at 18:00 and turned of 6:00 of the day after, from December, 2006 to December, 2007, coming down 52 samples. Weekly 287 species that were evaluated by using faunistic analysis of dominance, frequency, constancy and abundance, with 30 species distinguishing in these indexes. Astaena sp with 3.313 specimen collected in 44 from the 52 samples, Cyclocephala sp2 with 2415 collected in 51 from the 52 samples and Linepithema humile with 2299 in 18 samples.
\end{abstract}

Key words: Light traps, scarabaeidae, predominant, coleoptera

\footnotetext{
1 Parte da dissertação do primeiro autor apresentada ao Programa de Pós-Graduação em Agronomia, PPGA da Universidade Federal do Piauí para obtenção do título de Mestre em Agronomia.

2 Pós-Graduando do Programa de Pós-Graduação em Agronomia da Universidade Federal do Piauí. E-mail: rommeltito@bol.com. br; gilsonportela@uol.com.br; ocimaralencar@hotmail.com.br

3 Prof. do Departamento de Fitotecnia, PPGA/CCA, Universidade Federal do Piauí- UFPI, Campus do Socopo, CEP 64.049-550, Teresina, PI. E-mail: pramalhoufpi@yahoo.com.br; lempadua@ufpi.br

* Autor para correspondência
} 


\section{Introdução}

A cana-de-açúcar é uma das culturas agrícolas mais importantes da região tropical, gerando centenas de milhares de empregos diretos e indiretos, o que a torna uma importante fonte de renda e desenvolvimento. No Brasil, a cultura da cana-deaçúcar é considerada uma das mais importantes, tanto pela área que ocupa, como do ponto de vista econômico, ambiental e social (URQUIAGA et al., 1991). No entanto esta cultura apresenta um grande número de insetos-pragas, podendo ultrapassar 1.500 espécies em todo o mundo (LONG; HENSLEY, 1972).

As pragas que proporcionam os maiores prejuízos na cultura da cana são as brocas do colmo Lepidoptera (Crambidae), do gênero Diatraea saccharalis (Fabricius, 1794) (MACEDO; ARAÚJO, 2000). O principal prejuízo é causado pela ação de fitopatógenos, como o Fusarium moniliforme e Colletotrichum falcatum, que penetram pelos orifícios feitos pelas lagartas, causadores das podridões de fusário e podridãovermelha, respectivamente, responsáveis pela inversão e perda de sacarose.

Também de igual importância, porém com distribuição bem mais restrita, é a broca gigante Castnia licus, que tem provocado prejuízos que podem ultrapassar $60 \%$ em muitos canaviais do Nordeste (NAKANO et al., 2002).

Vale ressaltar as cigarras Mahanarva fimbriolata e M. posticata, que vêm ganhando importância como praga, devido à prática cada vez mais freqüente do corte da cana crua (sem queima), (BATISTA FILHO et al., 2003).

Os canaviais servem de abrigo a inúmeros insetos, sendo que se encontram inseridos nesta comunidade os predadores que se alimentam tanto dos insetos-pragas como dos que não são pragas (MACEDO; ARAÚJO, 2000).

Este é um trabalho pioneiro e de relevante necessidade, pois apesar da cultura canavieira estar instalada no Estado do Piauí há aproximadamente 30 anos, não existem pesquisas com entomofauna associada à cana-de-açúcar, tornando este, por conseguinte, o primeiro registro de espécies de insetos associados à cultura da cana-de-açúcar no Estado do Piauí, o que servirá de base para projetos futuros, em especial a programas de manejo ecológico de pragas relacionados à cultura. Sendo assim este trabalho teve como objetivo estudar a entomofauna associada à cultura da cana-de-açúcar, no município de União (PI), através de análise faunística.

\section{Material e Métodos}

Local de coleta

$\mathrm{O}$ experimento foi instalado em área comercial de cana-de-açúcar, no município de União (PI). O município apresenta formação vegetal típica de Mata de Cocal, que se caracteriza por ser uma área de transição entre floresta amazônica e cerrado, caracterizada por predomínio de côco babaçu (Orbignya phalerata). A usina cultiva comercialmente as variedades SP73-2577, SP832847, SP81-3250, SP79-1011 e RB92-579, a idade do canavial vai desde cana planta até cana com idade de 04 anos, o clima é seco a maior parte do ano e na área existem grande número de bolsões de mata nativa (áreas de preservação permanente) ao longo de toda sua extensão.

Coleta e identificação dos insetos

As coletas foram efetuadas semanalmente, utilizando armadilha luminosa, modelo "Luiz de Queiroz” (SILVEIRANETO et al., 1976), provida de lâmpadas fluorescentes, ultravioleta, modelo F15 T8 $\mathrm{BL}$, posicionada na altura de $1,5 \mathrm{~m}$, nas coordenadas geográficas $04^{\circ} 51^{\prime} 08^{\prime \prime} \mathrm{S}$ e $42^{\circ} 52^{\prime} 59^{\prime \prime} \mathrm{W}$. A armadilha era ligada uma vez por semana, às 18:00 horas e desligada às 06:00 horas do dia seguinte, no período de dezembro de 2006 a dezembro de 2007, perfazendo um total de 52 coletas.

Os insetos capturados eram colocados em sacos 
plásticos etiquetados e levados ao Departamento de Fitotecnia do Centro de Ciências Agrárias da Universidade Federal do Piauí (CCA/ UFPI), para triagem. Cada táxon foi numerado, quantificado e montado em alfinete entomológico e, em seguida, acondicionado em caixa entomológica contendo naftalina.

A identificação dos taxa foi realizada pelo Dr. Sinval Silveira Neto, professor da Escola Superior de Agricultura "Luiz de Queiroz" - ESALQ/ USP, Campus de Piracicaba (SP). A entomofauna coletada foi identificada prioritariamente em nível de espécie ou gênero ou, pelo menos, em nível de família. Todos os espécimes estão depositados na Coleção de Entomologia do Laboratório de Fitossanidade do CCA-UFPI.

\section{Medidas da fauna}

Para efeito de cálculo, foram adotados os seguintes critérios: as espécies que surgiram de uma, duas, três ou quatro vezes em uma única coleta e duas ou três vezes em duas coletas foram descartadas das análises, por serem pouco freqüentes, raras, acidentais e não dominantes.

Após o descarte, as espécies restantes foram caracterizadas através do uso dos seguintes índices faunísticos: dominância, abundância, freqüência e constância. Os índices foram determinados através do software "ANAFAU” (MORAES et al., 2003), que calcula os valores, segundo Silveira Neto et al. (1976).

Os critérios utilizados pelo software para classificar os índices faunísticos foram os seguintes:

As espécies quanto a dominância são classificadas em: dominante (D) - freqüência maior que o limite da dominância e não-Dominante (ND) - freqüência menor que o limite da dominância.

A abundância foi classificada nas seguintes classes: rara $(\mathrm{R})$ - número de indivíduos menor que o limite inferior ao intervalo de confiança (IC) da média; dispersa (D) - número de indivíduos entre os limites inferior e superior do IC da média; comum (C) - número de indivíduos entre os limites inferior e superior do IC da média; abundante (A) - número de indivíduos entre os limites superiores do IC e muito abundante (MA) - número de indivíduos maior que o limite superior do IC da média.

As classes de freqüência foram: pouco freqüente (PF) - freqüência menor que o limite inferior do IC da média; freqüente $(F)$ - freqüência entre os limites inferior e superior do IC da média e muito freqüente (MF) - freqüência maior que o limite superior do IC da média.

Em relação a constância os taxa foram classificados conforme a seguir: constante (W) maior que o limite do IC; acessória $(\mathrm{Y})$ - número situado dentro do IC e acidentais $(\mathrm{Z})$ - menor que o limite inferior de IC.

\section{Resultados e Discussão}

\section{Levantamento}

$\mathrm{O}$ número total de indivíduos coletados em área de cana-de-açúcar no município de União (PI), durante o período de $12 / 2006$ a 12/2007, foi 259.561 insetos, distribuídos em 576 morfo-espécies, 374 gêneros e 132 famílias, pertencentes a 16 ordens. Deste total, 233.176 indivíduos pertenciam a uma espécie de díptero da família Dolichopodidae, o que representou $89,83 \%$ do total dos insetos capturados. A espécie se encontrava no local devido à presença de cursos de água que se formam durante a estação chuvosa e devido, também, à irrigação feita durante o período pós-corte.

A espécie Astaena sp foi a que apresentou maior númerodeindivíduos daordem coleoptera, com 3.313 presentes em 44 das 52 coletas realizadas (Tabela 1). Esta espécie surgiu como a mais representativa pela presença de irrigação com resíduo de vinhaça para elevar o teor de compostos nitrogenados e pela presença de tanques de vinhaça nas proximidades, pois segundo Costa Lima (1953) e Almeida Filho 
(1995), todas as espécies pertencentes à sub-família Aphodinae são de natureza detritiva e incorporadoras de resíduos orgânicos, tanto na fase jovem como adulta. Após o descarte, restaram 25.961 indivíduos, distribuídos em 287 taxa, conforme a (Tabela 2).
Caracterização da Comunidade

\section{Dominância}

A distribuição dos taxa coletados no município de União (PI), com relação à dominância, pode ser observada através da (Tabela 2).

Tabela 1. Distribuição de indivíduos por coletas com relação aos índices faunísticos dominância, abundância, freqüência e constância e hábitos alimentares, capturados em área de cana-de-açúcar, no município de União (PI), de dezembro de 2006 a dezembro de 2007.

\begin{tabular}{|c|c|c|c|c|c|c|c|}
\hline ESPÉCIE & $\mathrm{N}^{\mathrm{o}}$ IND. & $\mathrm{N}^{\circ}$ COLETAS & DOMIN. & ABUND. & FREQ. & CONST. & HÁBITO \\
\hline Acromyrmex sp $^{1}$ & 156 & 08 & $\mathrm{D}$ & MA & $\mathrm{F}$ & $\mathrm{Y}$ & Carnívoro \\
\hline Astaena sp & 3.313 & 44 & $\mathrm{D}$ & MA & MF & W & Herbívoro \\
\hline Ataenus $\mathrm{sp}^{1}$ & 551 & 17 & $\mathrm{D}$ & MA & MF & $\mathrm{W}$ & Herbívoro \\
\hline Belostoma sp & 93 & 16 & $\mathrm{D}$ & $\mathrm{C}$ & $\mathrm{F}$ & W & Predador \\
\hline Braconidae $\mathrm{sp}^{1}$ & 125 & 10 & $\mathrm{D}$ & $\mathrm{C}$ & $\mathrm{F}$ & W & Parasitoide \\
\hline Brasiela sp & 164 & 18 & $\mathrm{D}$ & MA & MF & $\mathrm{W}$ & Predador \\
\hline Buenoa sp & 590 & 36 & $\mathrm{D}$ & MA & MF & $\mathrm{W}$ & Predador \\
\hline Buprestidae $\mathrm{sp}^{2}$ & 303 & 12 & $\mathrm{D}$ & MA & MF & W & Herbívoro \\
\hline Camponotus $\mathrm{sp}^{2}$ & 203 & 22 & $\mathrm{D}$ & MA & MF & $\mathrm{W}$ & Predador \\
\hline Clivina $\mathrm{sp}^{1}$ & 160 & 27 & $\mathrm{D}$ & MA & MF & W & Predador \\
\hline Clivina $\mathrm{sp}^{3}$ & 90 & 15 & ND & $\mathrm{C}$ & $\mathrm{F}$ & W & Predador \\
\hline Coleoptera $\mathrm{sp}^{1}$ & 788 & 30 & $\mathrm{D}$ & MA & MF & W & Herbívoro \\
\hline Culex sp & 263 & 15 & $\mathrm{D}$ & MA & MF & $\mathrm{W}$ & Herbívoro \\
\hline Cyclocephala $\mathrm{sp}^{1}$ & 558 & 43 & $\mathrm{D}$ & MA & MF & W & Herbívoro \\
\hline Cyclocephala $\mathrm{sp}^{2}$ & 2.415 & 51 & $\mathrm{D}$ & MA & MF & $\mathrm{W}$ & Herbívoro \\
\hline Diatraea saccharalis & 348 & 28 & $\mathrm{D}$ & MA & MF & $\mathrm{W}$ & Herbívoro \\
\hline Formicidae $\mathrm{sp}^{5}$ & 113 & 14 & $\mathrm{D}$ & $\mathrm{C}$ & $\mathrm{F}$ & $\mathrm{W}$ & Predador \\
\hline Formicidae $\mathrm{sp}^{2}$ & 222 & 16 & $\mathrm{D}$ & MA & MF & $\mathrm{W}$ & Predador \\
\hline Formicidae $\mathrm{sp}^{4}$ & 215 & 12 & $\mathrm{D}$ & MA & MF & $\mathrm{W}$ & Predador \\
\hline Formicidae $\mathrm{sp}^{8}$ & 372 & 13 & $\mathrm{D}$ & MA & MF & $\mathrm{W}$ & Predador \\
\hline Gyrinidae $\mathrm{sp}^{1}$ & 663 & 40 & $\mathrm{D}$ & MA & MF & $\mathrm{W}$ & Predador \\
\hline Gyrinidae $\mathrm{sp}^{2}$ & 352 & 27 & $\mathrm{D}$ & MA & MF & $\mathrm{W}$ & Predador \\
\hline Gyrinidae $\mathrm{sp}^{3}$ & 149 & 14 & $\mathrm{D}$ & MA & MF & $\mathrm{W}$ & Predador \\
\hline Gyrinidae $\mathrm{sp}^{4}$ & 627 & 37 & $\mathrm{D}$ & MA & MF & $\mathrm{W}$ & Predador \\
\hline Gyrinus $\mathrm{sp}^{1}$ & 1.300 & 38 & $\mathrm{D}$ & MA & MF & $\mathrm{W}$ & Predador \\
\hline Hydrophilidae $\mathrm{sp}^{2}$ & 472 & 14 & $\mathrm{D}$ & MA & MF & $\mathrm{W}$ & Predador \\
\hline Ichneumonidae $\mathrm{sp}^{2}$ & 95 & 20 & $\mathrm{D}$ & $\mathrm{C}$ & $\mathrm{F}$ & $\mathrm{W}$ & Predador \\
\hline Lineptema humili & 2.299 & 18 & $\mathrm{D}$ & MA & MF & $\mathrm{W}$ & Predador \\
\hline Lygaeidae $\mathrm{sp}^{2}$ & 104 & 22 & $\mathrm{D}$ & $\mathrm{C}$ & $\mathrm{F}$ & $\mathrm{W}$ & Herbívoro \\
\hline Melyriedae $\mathrm{sp}^{2}$ & 181 & 7 & $\mathrm{D}$ & MA & MF & $\mathrm{Z}$ & Herbívoro \\
\hline Metasidae $\mathrm{sp}^{2}$ & 178 & 14 & $\mathrm{D}$ & MA & MF & $\mathrm{W}$ & Herbívoro \\
\hline Nasutiterme sp & 143 & 07 & $\mathrm{D}$ & MA & MF & $\mathrm{Z}$ & Herbívoro \\
\hline Neotridactylus $\mathrm{sp}^{1}$ & 179 & 24 & $\mathrm{D}$ & MA & MF & $\mathrm{W}$ & Herbívoro \\
\hline
\end{tabular}




\begin{tabular}{lccccccc}
\hline ESPÉCIE & $\mathrm{N}^{\circ}$ IND. & $\mathrm{N}^{\circ}$ COLETAS & DOMIN. & ABUND. & FREQ. & CONST. & HÁBITO \\
\hline Neotridactylus sp & 107 & 16 & $\mathrm{D}$ & $\mathrm{C}$ & $\mathrm{F}$ & $\mathrm{W}$ & Herbívoro \\
Notiobia sp & 142 & 14 & $\mathrm{D}$ & $\mathrm{MA}$ & $\mathrm{MF}$ & $\mathrm{W}$ & Predador \\
Pangaeus sp & 238 & 25 & $\mathrm{D}$ & $\mathrm{MA}$ & $\mathrm{MF}$ & $\mathrm{W}$ & Herbívoro \\
Platypus sp & 233 & 44 & $\mathrm{D}$ & $\mathrm{MA}$ & $\mathrm{MF}$ & $\mathrm{W}$ & Herbívoro \\
Polymetarcidae sp & 80 & 23 & $\mathrm{ND}$ & $\mathrm{C}$ & $\mathrm{F}$ & $\mathrm{W}$ & Predador \\
Scaptocores castanea & 81 & 09 & $\mathrm{ND}$ & $\mathrm{C}$ & $\mathrm{F}$ & $\mathrm{Y}$ & Herbívoro \\
Selenophorus sp & 324 & 38 & $\mathrm{D}$ & $\mathrm{MA}$ & $\mathrm{MF}$ & $\mathrm{W}$ & Predador \\
Selenophorus sp & 190 & 16 & $\mathrm{D}$ & $\mathrm{C}$ & $\mathrm{F}$ & $\mathrm{W}$ & Predador \\
Tagosode orezicola & 126 & 22 & $\mathrm{D}$ & $\mathrm{C}$ & $\mathrm{F}$ & $\mathrm{W}$ & Herbívoro \\
Tenagobia sp & 137 & 05 & $\mathrm{D}$ & $\mathrm{A}$ & $\mathrm{MF}$ & $\mathrm{Z}$ & Herbívoro \\
Tenebrionidae sp & 583 & 09 & $\mathrm{D}$ & $\mathrm{MA}$ & $\mathrm{MF}$ & $\mathrm{Y}$ & Herbívoro \\
Tropisternos colaris & 251 & 29 & $\mathrm{D}$ & $\mathrm{MA}$ & $\mathrm{MF}$ & $\mathrm{W}$ & Predador \\
Tropisternos laevis & 1.785 & 27 & $\mathrm{D}$ & $\mathrm{MA}$ & $\mathrm{MF}$ & $\mathrm{W}$ & Predador \\
\hline
\end{tabular}

$\mathrm{D}=$ dominantes $/ \mathrm{ND}=$ não dominantes $/ \mathrm{MA}=$ muito abundante $/ \mathrm{A}=$ abundante $/ \mathrm{C}=$ comum $/ \mathrm{D}=$ dispersa $/ \mathrm{R}=\mathrm{rara} / \mathrm{MF}=\mathrm{muito}$ freqüente $/ \mathrm{F}=$ freqüente $/ \mathrm{PF}=$ pouco freqüente $/ \mathrm{W}=$ constante $/ \mathrm{Y}=$ acessório $/ \mathrm{Z}=$ acidental

Tabela 2. Distribuição percentual dos taxa com relação aos índices faunísticos: dominância, abundância, freqüência e constância, capturados em área de cana-de-açúcar, no município de União (PI), de dezembro de 2006 a dezembro de 2007.

\begin{tabular}{|c|c|c|c|c|c|}
\hline $\begin{array}{l}\text { ÍNDICE } \\
\text { FAUNÍSTICO }\end{array}$ & CLASSIFICAÇÃO & $\begin{array}{l}\text { NÚMERO DE } \\
\text { TAXA }\end{array}$ & $\%$ DE TAXA & $\begin{array}{l}\text { NÚMERO DE } \\
\text { INDIVÍDUOS }\end{array}$ & $\begin{array}{c}\% \text { DE } \\
\text { INDIVÍDUOS }\end{array}$ \\
\hline \multirow{3}{*}{ Dominância } & $\mathrm{D}$ & 43 & 14,63 & 20.968 & 80,76 \\
\hline & ND & 244 & 85,37 & 4.993 & 19,23 \\
\hline & MA & 34 & 11,84 & 20.594 & 79,32 \\
\hline \multirow{4}{*}{ Abundância } & A & 01 & 0,35 & 137 & 0,52 \\
\hline & $\mathrm{C}$ & 29 & 10,10 & 2.268 & 8,73 \\
\hline & $\mathrm{D}$ & 11 & 3,83 & 529 & 2,03 \\
\hline & $\mathrm{R}$ & 212 & 73,87 & 2.433 & 9,37 \\
\hline \multirow{3}{*}{ Frequência } & MF & 35 & 12,19 & 20.862 & 80,36 \\
\hline & $\mathrm{F}$ & 29 & 10,10 & 2.273 & 8,75 \\
\hline & PF & 223 & 70,70 & 2.826 & 10,88 \\
\hline \multirow{3}{*}{ Constância } & W & 81 & 28,28 & 22.380 & 86,20 \\
\hline & $\mathrm{Y}$ & 17 & 5,92 & 1.196 & 4,60 \\
\hline & Z & 189 & 65,85 & 2.385 & 9,18 \\
\hline
\end{tabular}

$\mathrm{D}=$ dominantes $/ \mathrm{ND}=$ não dominantes $/ \mathrm{MA}=$ muito abundante $/ \mathrm{A}=$ abundante $/ \mathrm{C}=$ comum $/ \mathrm{D}=$ dispersa $/ \mathrm{R}=$ rara $/ \mathrm{MF}=$ muito freqüente $/ \mathrm{F}=$ freqüente $/ \mathrm{PF}=$ pouco freqüente $/ \mathrm{W}=$ constante $/ \mathrm{Y}=$ acessório $/ \mathrm{Z}=$ acidental. 
Os taxa dominantes representaram apenas $14,98 \%$ de um total de 287, sendo que estes 43 taxa totalizaram $80,76 \%$ dos indivíduos coletados (Tabela 2), o que pode ser explicado em função do número elevado de Astaena sp, com 3.313 indivíduos. Tal fato contribuiu para que um pequeno número de taxa fosse categorizado como dominantes. Fato semelhante foi registrado nos trabalhos realizados por Almeida Filho (1995) e por Lutinski e Garcia (2005), que encontraram um pequeno número de taxa dominante, contendo uma grande porcentagem do total de indivíduos coletados.

\section{Abundância}

Com relação à abundância (Tabela 2), os taxa muito abundantes apareceram representados por apenas $11,84 \%$ do total. Porém, em número de indivíduos, somaram $79,32 \%$ dos indivíduos coletados, concordando com Almeida Filho (1995), que realizou experimento em cana crua e cana queimada, no município de Piracicaba (SP) e com Canettieri e Garcia (2000), que estudaram a abundância relativa de espécies de Cerambycidae, no município de Ceres (GO) e verificaram que um pequeno número de taxa muito abundantes apresentou a maioria das espécimes coletadas.

Apenas a taxa (Tenagobia sp) foi considerado abundante, correspondendo a apenas $0,52 \%$; já os taxa comuns representaram $8,73 \%$ do total, com 2.268 espécimes. Ainda segundo este índice faunístico, os taxa dispersos tiveram representatividade baixa igual a 2,03\%; já os taxa raros, embora somassem 212 do total de 287 em porcentagem de espécimes, representaram apenas $9,37 \%$.

\section{Freqüência}

Verificou-se que os muito freqüentes (Tabela 2) foram os mais representativos, com $80,36 \%$ do total de insetos coletados, embora totalizassem 35 taxa; 29 taxa foram considerados freqüentes, sendo, porém, correspondentes a apenas $8,75 \%$ do total de insetos; já os pouco freqüentes, embora tenham predominado em número de taxa com 223 , corresponderam apenas a 10,88\% dos indivíduos coletados.

Comportamento semelhante, com relação ao índice freqüência, também foi verificado por Viana e Costa (2001) com lepidópteros em duas comunidades florestais, em Itaára (RS), em que poucos taxa muito freqüentes apresentaram maioria do total de espécies coletadas.

\section{Constância}

Com relação ao índice constância (Tabela 2), foram considerados constantes 81 taxas, representando $86,20 \%$ do total e apresentando 22.380 insetos; foram considerados como acessórios 17 taxa, o que representou $4,60 \%$ da entomofauna coletada; por outro lado, os taxa acidentais, embora tenham surgido 189 vezes, com $9,18 \%$ do total de 25.961 insetos coletados, foram pouco significativos quando comparados aos constantes. Comportamento semelhante foi verificado por Lutinski e Garcia (2005), que realizaram análise faunística de formigas em Chapecó (SC), onde encontraram maioria das espécies distribuídas em pequeno número de taxa.

\section{Predominância}

Com relação à predominância, $10,45 \%$ do total dos taxa coletados se sobressaíram nos quatro índices faunísticos utilizados para classificar os insetos, totalizando 19.657 indivíduos. As espécies que mais contribuíram para este elevado número de indivíduos foram Astaena SP e Cyclocephala sp2 (Scarabaeidae), Linepithema humile (Formicidae), Gyrinus SP (Gyrinidae) e Tropisternus leavis (Hydrophilidae) (Tabela 01), que somaram 11.112 indivíduos, perfazendo $42,83 \%$ do total coletado.

Ao comparar os resultados encontrados nesta pesquisa com os resultados encontrados por Almeida Filho (1995), em Piracicaba (SP), verificou-se que 
$20 \%$ dos taxa em nível de gênero foram coletados tanto em União (PI) como em Piracicaba (SP), sendo eles: Diatraea, Pangaeus, Cyclocephala, Selenophorus, Clivina e Tropisternus. Tal resultado demonstrou que mesmo a entomofauna das duas regiões diferindo em $80 \%$, ainda assim existem espécies comuns que são de importância econômica. Quando os resultados encontrados nesta pesquisa são comparados com os resultados obtidos por Macedo e Araújo (2000), em levantamento de predadores feito em canavial de Piracicaba (SP), que apareceram os gêneros Lebia, Selenophorus, Arthrostyctus, Clivina, Eciton, Doru e Conoderus como os mais representativos. Este resultado demonstrou que Selenophorus e Clivina foram representativos tanto no canavial em Piracicaba (SP) quanto nesta pesquisa. Confrontado os resultados obtidos nesta pesquisa com os obtidos por Silveira Neto et al. (1995), em levantamento de entomofauna no Departamento de Entomologia da ESALQ/ USP, verifica-se que apenas 6,66 dos taxa, em nível de gênero, foram coincidentes, diferindo bastante a entomofauna das duas regiões.

\section{Conclusões}

A entomofauna encontrada no município de União (PI) apresenta um grande número de espécies e gêneros distribuídos em muitas ordens.

Agrande biodiversidade da entomofauna revelada neste trabalho provavelmente deve-se à presença de áreas de preservação em meio ao canavial.

Os insetos predominantes coletados no município deUnião(PI) encontram-se distribuídos nas seguintes famílias: Crambidae, Hydrophilidae, Culicidae, Formicidae, Scarabaeidae, Gyrinidae, Notonectidae, Cydnidae, Carabidae, Cicindelidae, Metasidae, Buprestidae, Platypodidae, Tridactylidae.

\section{Referências}

ALMEIDA FILHO, A. J. de. Impacto ambiental da queima da cana-de-açúcar sobre a entomofauna. 1995. (Doutorado em Ciência Biológica) - Escola Superior de Agricultura Luiz de Queiroz, Piracicaba.

BATISTA FILHO, A.; ALMEIDA, J. E. M.; SANTOS, A. S.; MACHADO, L. A.; ALVES, S. B. Eficiência de isolamento de Metarhizium anisopliae no controle de cigarrinha-da-raiz da cana-de-açúcar (Mahanarva fimbriolata) (Hom. Cercopidae). Arquivo do Instituto Biológico, São Paulo, v. 70, n. 3, p. 309-314, 2003.

CANETTIERI, E. R. P. D. A. S.; GARCIA, A. H. Abundância relativa das espécies de Cerambycidae (Insecta-Coleoptera) em pomar de frutíferas misto. Pesquisa Agropecuária Tropical, Goiás, v. 30, n. 2, p. 43-50, 2000.

COSTA LiMA, A. M. da. Insetos do Brasil. Coleópteros. Tomo 8. Rio de Janeiro: Escola Nacional de Agronomia do Rio de Janeiro, 1953. 323 p. (Série Didática, 10).

LONG, W. H.; HENSLEY, S. D. Insect pests of sugar cane. Annual Reviews, v. 17, p. 149-176. 1972. Disponível em: <http.//www.annualreviews.org/aronline>. Acesso em: 15 jan. 2008.

LUTINSKI, J. A.; GARCIA, F. R. M. Análise faunística de formigas em ecossistema degradado no município de Chapecó, SC. Revista Biotemas, Florianópolis, v. 18, n. 2, p. 73-86, 2005.

MACEDO, N.; ARAÚJO, E. J. R. Efeitos da queima do canavial sobre insetos predadores. Anais da Sociedade Entomológica do Brasil, Araras, v. 29, n. 1, p. 71-77, 2000.

MORAES, R. C. B.; HADDAD, M. L.; SILVEIRANETO, S.; REYES, A. E. L. Software para análise faunística. In: SIMPÓSIO DE ENCONTRO BIOLÓGICO, 8., 2003, São Pedro. Anais... São Pedro: Sinpobiol, p. 195, 2003.

NAKANO, O.; SILVEIRA NETO, S.; CARVALHO, R. P. L.; BAPTISTA, G. C.; BERTI FILHO, E.; PARRA, J. R. P.; ZUCCHI, R. A.; ALVES, S. B.; VENDRAMIM, J. D.; MARCHINI, L. C.; LOPES, J. R. S.; OMOTO, C.; GALLO, D. Entomologia agrícola. Piracicaba: FEALQ, v. $10,2002,920$ p.

SILVEIRA NETO, S.; MONTEIRO, R. C.; ZUCCHI, R. A.; MORAES, R. C. B. Uso da análise faunística de insetos na avaliação do impacto ambiental. Scientia Agricola, Piracicaba, v. 52, n. 1, p. 09-15, 1995. 
SILVEIRA NETO, S.; NAKANO, O.; BARBIN, D.; VILLA NOVA, N. Manual de ecologia dos insetos. São Paulo: Ceres, 1976. 419 p.

URQUIAGA, S.; BODDEY, R. M.; OLIVEIRA, O. C. de; LIMA, E.; GUIMARÃES, D. H. V. A importância de não queimar a palha na cultura da cana-de-açúcar. Seropédica: Ministério da Agricultura, do Abastecimento e da Reforma Agrária/ EMBRAPA-CNPBS. 1991, 6 p. (Circular técnica, n. 5).

VIANA, T. M. B.; COSTA, E. C. Lepidópteros associados a duas comunidades florestais em Itaára, RS. Revista Ciência Florestal, Santa Maria, v. 11, n. 1, p. 6780, 2001. 